\title{
Adaptive Access Class Barring Method for Machine Generated Communications
}

\author{
Jaesung Park ${ }^{1}$ and Yujin Lim² \\ ${ }^{1}$ Department of Information Security, University of Suwon, San 2-2, Wau-ri, Bongdam-eup, Hwaseong, \\ Gyeonggi-do 445-743, Republic of Korea \\ ${ }^{2}$ Department of Information Technology Engineering, Sookmyung Women's University, Cheongpa-ro 47-gil 100, \\ Yongsan-gu, Seoul 04310, Republic of Korea
}

Correspondence should be addressed to Yujin Lim; yujin91@sookmyung.ac.kr

Received 10 June 2016; Accepted 27 July 2016

Academic Editor: Youngwook Ko

Copyright (C) 2016 J. Park and Y. Lim. This is an open access article distributed under the Creative Commons Attribution License, which permits unrestricted use, distribution, and reproduction in any medium, provided the original work is properly cited.

\begin{abstract}
Cellular network is provisioned to serve traffic demands generated by human being. The random access channel used for nodes to compete for a connection with an eNB is limited. Even though machines generate very small amount of data traffic, the signaling channel of a network becomes overloaded and collisions occur to fail the access if too many MTC (Machine Type Communication) devices attempt to access network. To tackle the issue, 3GPP specifies an access class barring but leaves a specific algorithm as an implementation issue. In this paper, we propose an adaptive access barring method. Generally, an eNB does not know the number of MTC devices in its coverage area. Thus, it is difficult to control the barring factor by predicting the number of MTC devices in a service area of a cell. On the contrary, we control the barring factor based on the prediction of access intensity which can be measured at an eNB. Simulation results show that since the proposed method can manipulate the barring factor autonomously according to the access intensity, it is superior to the original method in terms of the access success probability and the collision probability.
\end{abstract}

\section{Introduction}

MTC (Machine Type Communication) application is one of automated applications which provide connectivity between a tremendous number of embedded devices in LTE-A (Long Term Evolution-Advanced) [1, 2]. These embedded devices support a wide range of applications such as smart grid/smart metering, e-health, surveillance and security, and consumer electronics. Industry reports expect the significant potential of MTC applications to generate considerable revenues. In MTC, a large number of devices generate a huge amount of signaling or data flow to access RAN (Random Access Network) in a short time. Concurrent accesses from a large number of devices result in significant congestion. The system suffers from packet collision, long access delay, or low service quality of application. To alleviate the congestion, system needs to control the excessive connectivity from devices [3]. Thus, self-adaptation is a key building block of MTC application because it adaptively controls the strategic parameters to maximize the system performance in dynamic environment.
When a device is activated to send its data, it tries to reserve uplink contention-based channels, that is, RACHs (Random Access CHannels). In LTE-A, multichannel slotted ALOHA systems are used to model the random access channel. The two kinds of random access traffic arises from MTC devices: the traffic which is occurred by an external event and the traffic which periodically occurred to communicate between a device and a system in a synchronized manner. For example, for electricity meter reading, a device needs to report periodically electricity usage.

When a device tries to send data, it follows a random access 4-step procedure to reserve uplink channel in Figure 1. Firstly, a device selects a preamble randomly from a set of random access preambles. The selected preamble is transmitted to allow eNB to estimate the transmission timing of a device. In the second step, RAR (Random Access Response) is sent by eNB. It involves a timing advance command to adjust a device transmit timing and assigns uplink resources to a device. In the third step, a device transmits a message with identity information. If a preamble collision occurred in the first 


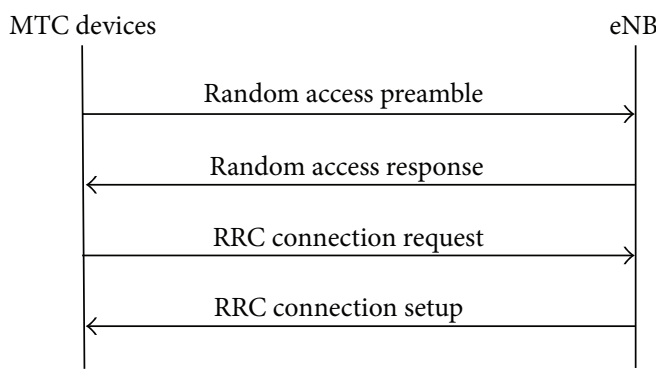

Figure 1: The random access procedure.

step, the colliding devices transmit identity information within the same time-frequency resource block. In this case, collision occurs at eNB. In the final step, eNB transmits a contention-resolution message. If packet collision occurs and eNB decodes one of collided packets, eNB acknowledges the device whose packet is successfully received. Unacknowledged devices fail the random access in current access opportunity and they will try again in the next access opportunity.

Under slotted ALOHA scheme, if the number of devices is controlled to access a system at the same time, system performance can be improved. Thus, eNB needs to optimally maintain the number of devices which simultaneously access a system. 3GPP (3rd Generation Partnership Project) has discussed some solutions such as ACB (Access Class Barring) and EAB (Extended Access Barring) [4]. Using ACB, devices defer their random access requests with a probability $p$. In other words, eNB periodically broadcasts barring parameters in SIB (System Information Block). Barring parameters involve a barring factor $(0 \leq p \leq 1)$ and a barring duration. Each device generates a random number between 0 and 1 and compares the number with $p$. If the random number is equal to or greater than $p$, the access is barred for the barring duration. In EAB, devices are classified into a set of ACs (Access Classes). An eNB periodically broadcasts SIB with a barring bitmap which indicates the barred ACs. When a device initiates a random access procedure, a device checks whether its AC is barred. The barred devices postpone their random access procedures until the next SIB is changed.

In $\mathrm{ACB}$ and $\mathrm{EAB}$, it is crucial to control the strategic parameters such as barring factor or barring bitmap. Even though many researchers try to solve the control problem, it is still open issue for further research [5]. Therefore, we propose a control method to determine a barring factor autonomously in ACB scheme to improve system performance. The experimental results show that our method improves system performances in terms of access success probability and collision probability.

The rest of the paper is organized as follows: in Section 2, we describe the related work. In Section 3, we present our control method to manipulate a barring factor in ACB. In Section 4, simulation results are summarized. In Section 5, conclusions and future research plan are drawn.

\section{Related Work}

There are $N$ orthogonal preambles in one random access opportunity (time slot). If more than one device send the same preamble in one slot, a collision happens. When a collision happens, a device tries again with a randomly chosen preamble in a new slot. As the number of devices increases, the congestion could be worse and system performance deteriorates.

To alleviate the congestion due to random access collision, control mechanisms have been proposed. Many mechanisms are presented to regulate the access opportunity of devices [6]. Backoff-based mechanisms distribute the access attempts from devices in time slots by using backoff window. Admission control-based mechanisms reject the access attempts based on the average admission rate [7]. ACBbased mechanisms distribute the access attempts based on the barring factor. It effectively reduces the collision probability under large transmissions at the same time. Among them, we focus on ACB-based mechanism to autonomously manipulate the barring factor. There is a trade-off between barring factor $p$ and LTE performance like resource utilization or average access delay. If the barring factor is smaller, fewer devices are allowed to access RACH. However, the access delay increases and resource utilization decreases. Thus, eNB needs to effectively control the barring factor under the current network condition. In [8], the barring factor is determined to maximize the expected number of successfully accessed devices in each slot. In [9], the factor is controlled to minimize the total service time which is the time for all devices to successfully access to eNB. In [10], an analytical model is proposed to minimize the access delay of all the devices. However, these mechanisms suffer from the high computational complexity to optimize the performance metrics. To reduce the complexity, approximation algorithms are also proposed to control the barring factor.

In an approximate approach, the current traffic load or predicted traffic load is mostly used to adaptively control the barring factor. In [11], the probability of packet transmission is calculated based on the current traffic load. Then, eNB determines the barring factor to improve network throughput. In [12], cooperative mechanism to control congestions among cells is proposed. eNBs cooperate to decide the barring factor in each cell for global stabilization and access load sharing. In [13], the number of contending devices is estimated using the Markov Chain with collision status. With the estimated load, the barring factor is determined. These conventional mechanisms estimate the number of contending devices by using traditional traffic prediction techniques.

\section{Proposed Method}

In this section, we describe an adaptive access barring method. The purpose of the method is to control the barring factor according to the estimated access intensity of MTC devices. The access intensity is defined as the number of random access attempts made during a certain time period.

An optimal predictor should know the exact correlation structure between the past measurements and the future 


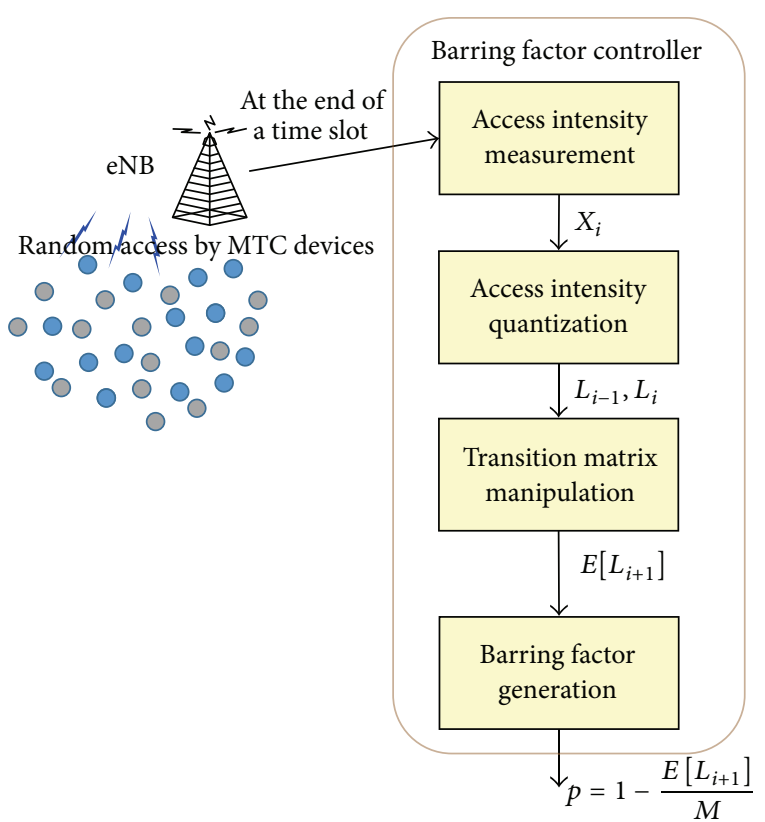

Silence MTC

- Triggered MTC

FIGURE 2: Barring factor controller.

measurements. However, it is not possible to know the future measurements. Thus, we design a practical predictor based on the past measurements. Figure 2 shows the block diagram of the barring factor controller. The controller is composed of four distinct modules: access intensity measurement module, access intensity quantization module, transition matrix manipulation module, and barring factor generation module.

The access intensity measurement module measures the access intensity during a certain time period. We assume that time is divided into a fixed time slot, the size of which is $t_{s}$. An eNB measures the access intensity during each time slot. We denote by $X_{i}$ the access intensity during the $i$ th time slot.

The access intensity quantization module discretizes the measured access intensity into $M$ access intensity levels. We denote by $A_{M}$ the maximum number of access intensities that a system allows. Then, $X_{i}$ is an integer and can be any value in $\left[0, A_{M}\right]$. However, as $A_{M}$ becomes larger, atomizing $X_{i}$ with the finest granularity demands more system resources for an eNB to maintain $X_{i}$ s for predicting future access intensity without improving system performance. For example, if $A_{M}$ is sufficiently large, $X_{i}=x$ and $X_{i}=x+1$ do not mean much to an eNB. Thus, we divide the range $\left[0, A_{M}\right]$ into $M$ access intensity levels. The height of each level is $\Delta=A_{M} / M$, where $M$ is an operating parameter controlling the granularity of $X_{i}$. We assign quantized values from $\Delta / 2$ to $(2 M-1) \Delta / 2$ to each access intensity level starting from the level corresponding to $[0, \Delta]$. Then, we approximate $X_{i}$ to the quantized value by a mapping function $L$. In other words, if we denote by $Q_{i}$ the quantized value of $X_{i}, Q_{i}=L\left(X_{i}\right)$. We also number the access intensity levels from zero to $M-1$ and denote by $L_{i}$ the access intensity level that $Q_{i}$ belongs to.
In the transition matrix manipulation module, the access intensity for the next time slot is estimated. An eNB maintains $M$ by $M+1$ state transition matrix $A[M][M+1]$. The transition matrix is used to approximate the correlation structure between $X_{i-1}$ and $X_{i}$. To achieve the goal, an eNB uses the transition matrix to keep track of the relation between the access intensity measured during the previous time slot $\left(Q_{i-1}\right)$ and the access intensity measured during the current time slot $\left(Q_{i}\right)$. In the state transition matrix, a state corresponds to the index number of an access intensity level. At the end of each time slot $i$, an eNB quantizes $X_{i-1}$ and $X_{i}$ into $Q_{i-1}=L\left(X_{i-1}\right)$ and $Q_{i}=L\left(X_{i}\right)$. Since $L_{i}$ is the access intensity level that $Q_{i}$ belongs to, the eNB updates the state transition matrix as follows:

$$
\begin{gathered}
A\left[L_{i-1}\right]\left[L_{i}\right]=A\left[L_{i-1}\right]\left[L_{i}\right]+1, \\
A\left[L_{i}\right][M+1]=A\left[L_{i}\right][M+1]+1 .
\end{gathered}
$$

Then, using the transition matrix, the transition matrix manipulation module predicts the access intensity level of the next time slot $i+1$ as

$$
E\left[L_{i+1} \mid L_{i}\right]=\sum_{j=1}^{M} j \operatorname{Pr}\left(L_{j} \mid L_{i}\right),
$$

where $\operatorname{Pr}\left(L_{i+1}=j \mid L_{i}\right)=A\left[L_{i}\right]\left[L_{j}\right] / A\left[L_{i}\right][M+1]$.

Using $E\left[L_{i+1} \mid L_{i}\right]$, the barring factor generation module determines the barring factor $p$ as follows:

$$
p=1-\frac{E\left[L_{i+1} \mid L_{i}\right]}{M} \text {. }
$$

\section{Performance Analysis}

We use the traffic model for smart electric metering MTC application as an experimental scenario [1]. Smart meters support various applications such as automatic meter reading, energy demand management, and microelectric generation management. In the traffic model, the household density in urban area of London is 35,670 households/cell. Periodical reporting of meter readings ranges from 5 mins to 24 hours [14]. The reporting cycle affects the access intensity of meters in a cell. We set reading frequency to 5 mins. In the experiments, we set $A_{M}$ to the maximum number of devices which attempt the random access in a time slot. For example, we set $A_{M}$ to 30 for the $100 \mathrm{~ms}$ time slot. The parameters for the random access channel of LTE-A networks follow [1].

An eNB serves a number of $N$ devices in a cell. Each device generates a single access request during a period of time $T$. In other words, all devices activate sending data between $t=0$ and $t=T$. In our experimental scenario, $T$ is set to 5 mins. The access intensity follows the distribution $p(t)$. The number of devices which generate access requests in the $i$ th access opportunity, that is, access intensity, is defined as follows:

$$
N_{\text {new }}^{i}=N \int_{t_{i}}^{t_{i+1}} p(t) d t
$$




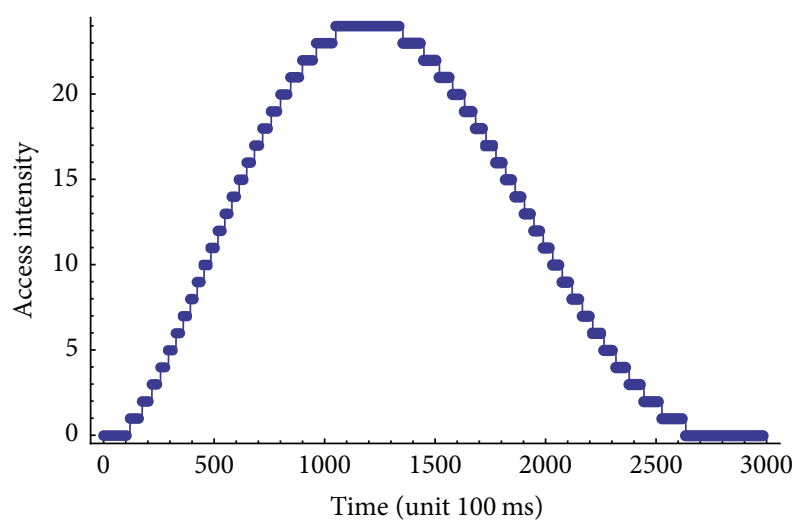

FIGURE 3: The access intensity of smart electric metering application.

where $t_{i}$ is the time of the $i$ th access opportunity and $p(t)$ follows the Beta distribution with the values of $\alpha=3$ and $\beta=4[1]$ :

$$
p(t)=\frac{t^{\alpha}(T-t)^{\beta-1}}{T^{\alpha+\beta-1} \operatorname{Beta}(\alpha, \beta)},
$$

where $\operatorname{Beta}(\alpha, \beta)$ is the Beta function. Figure 3 shows the access intensity for smart electric metering application.

We adopt the congestion coefficient to enable or disable a congestion control mechanism like ACB [15]. The congestion coefficient is the inverse of the ratio of the number of successful devices to the number of contending devices. When the congestion coefficient exceeds a specific threshold, the congestion control is activated. We set the threshold to 0.4 . It is deactivated when the congestion coefficient goes below the threshold. Once congestion control is activated, eNB manipulates the barring factor to regulate the access intensity.

Figure 4 shows the variance of barring factor with different number of access intensity levels $(M)$. We can see that the barring factor is adaptively controlled according to the access intensity. We analyze the variance of barring factor in in three aspects. The first is when the time slot is changed, $500 \mathrm{~ms}$ and $1000 \mathrm{~ms}$. The curve when the time slot is $1000 \mathrm{~ms}$ has less spikes than it when the time slot is $500 \mathrm{~ms}$. The second is when the number of access intensity levels is changed, 5 , 10,15 , and 20 . As the number of levels increases, the curves look like staircase. The last is when the access intensity within the traffic model is changed, increase period and decrease period. In the asymmetric curve of the Beta distribution in Figure 3, uphill slope is steeper than downhill slope. Thus, the curve of the barring factor in gently decreased period of access intensity is stable more than that in steeply increased period.

Figure 5 shows the variance of barring factor when the access intensity increases. In the figure, $N$ indicates 35,670 households/cell, that is, the household density in urban area of London. We set the number of access intensity levels to 10 . In steeply increased period of access intensity, our method rapidly reacts. In gently decreased period, the method slowly reacts. However, if the average of access intensity is too

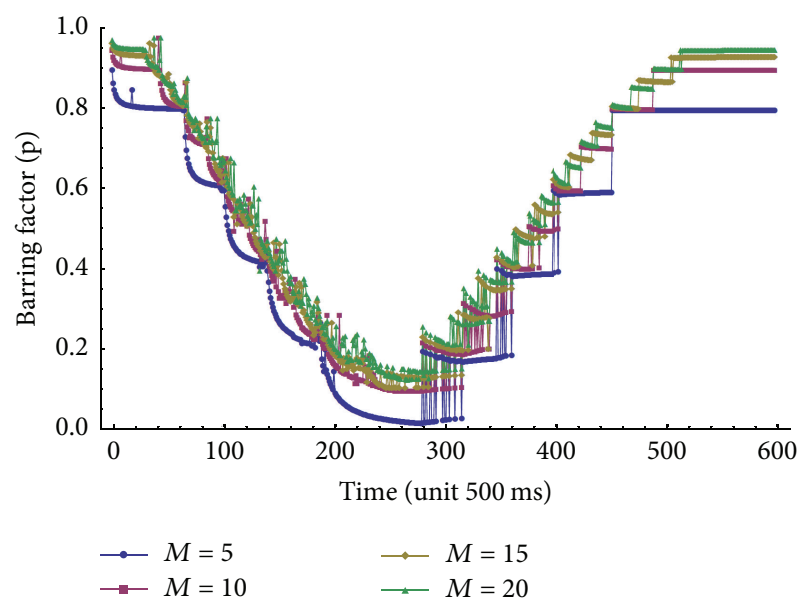

(a) Time unit $=500 \mathrm{~ms}$

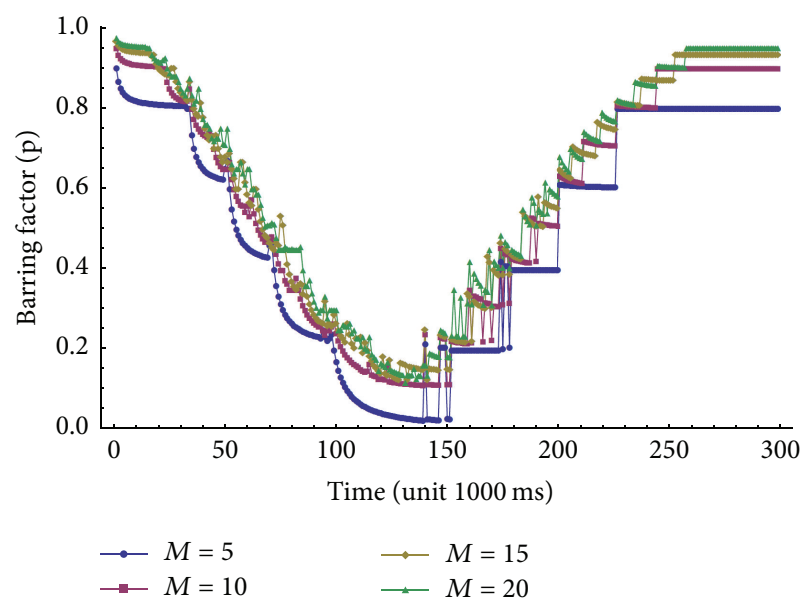

(b) Time unit $=1000 \mathrm{~ms}$

FIGURE 4: The value of barring factor by varying the number of access intensity levels.

low (in the figure, when the total number of devices = $0.5 \mathrm{~N}$ ), the colliding devices decrease and the barring factor correspondingly reacts.

Figure 6 shows the access success probability which is the successful completion probability of the random access in the maximum number of preamble transmissions. In the experiments, the time slots are changed from $60 \mathrm{~ms}$ to 200 ms. For comparison, we use the original ACB [4]. In the original $\mathrm{ACB}$, once the congestion control is activated, the barring factor is set to 0.1. When our method is used, the average of success probability is about $18 \%$ better than that of the original ACB. Figure 7 shows collision probability which is the ratio between the number of occurrences when two or more devices with the same preamble try to access RACH and the total number of access opportunities. When our method is used, the average of collision probability is about $12 \%$ better than that of the original ACB.

Figures 6 and 7 show that there is an optimal range on performance when $M$ is between 3 and 4 . As shown in Figure 8, when $M$ increases, the difference of access intensities between adjacent levels decreases and the effect of 


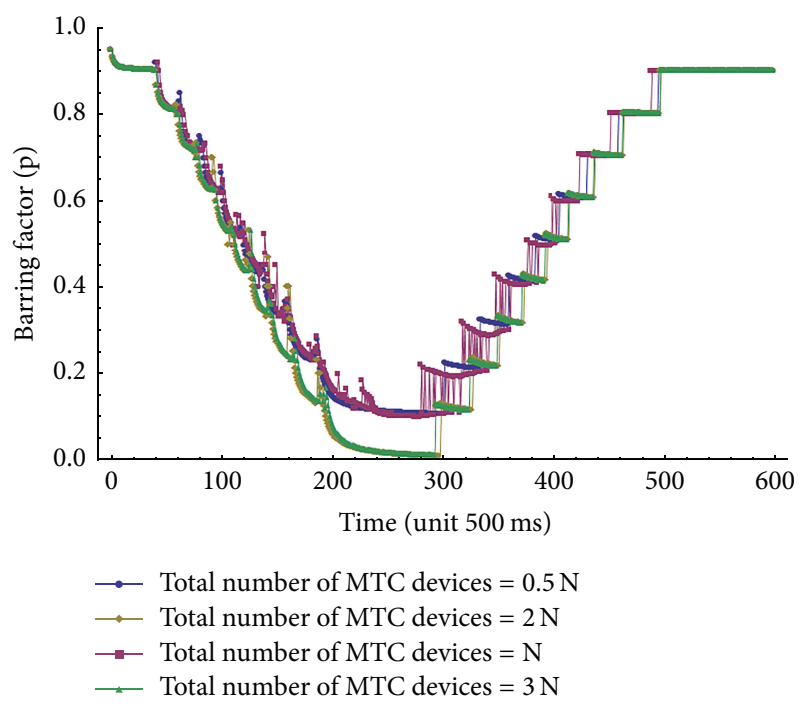

(a) Time unit $=500 \mathrm{~ms}$

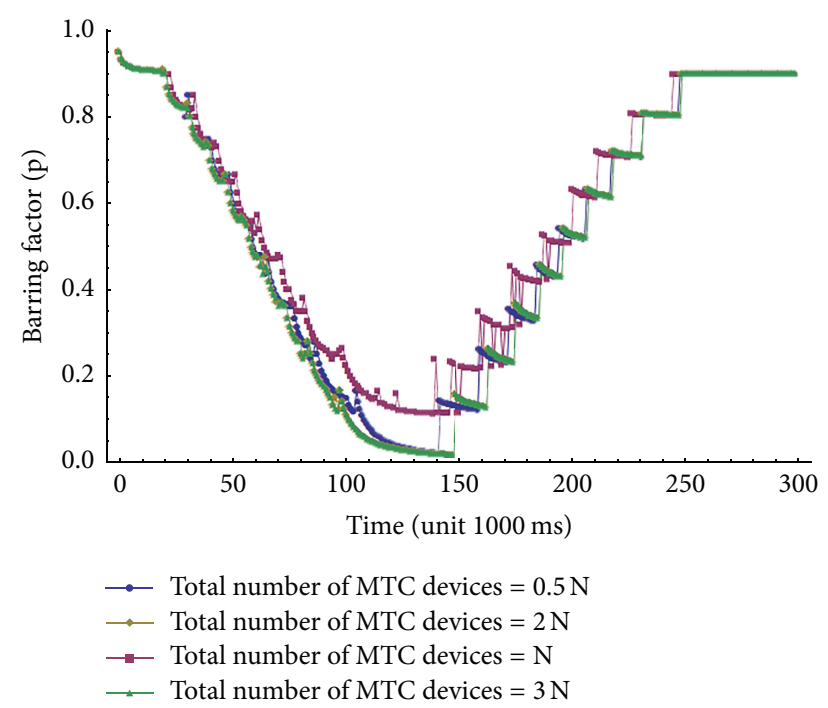

(b) Time unit $=1000 \mathrm{~ms}$

FIGURE 5: The value of barring factor by varying the number of devices.

changing the barring factor decreases. When $M$ decreases, the difference of access intensities between adjacent levels increases and the changing the access intensity does not finely affect the barring parameter adjustment. It indicates that the performance can be improved by controlling the number of access intensity levels.

Figure 9 shows the variance of the number of successful devices for each time slot. The successful device indicates the device which successfully accesses RACH in a time slot. When the access intensity is low, the performances of our method and the original ACB are similar. However, when the access intensity is high, the number of successful devices in our method is 3.5 times larger than that in the original ACB. In other words, our method efficiently controls the barring factor to improve the system performance.

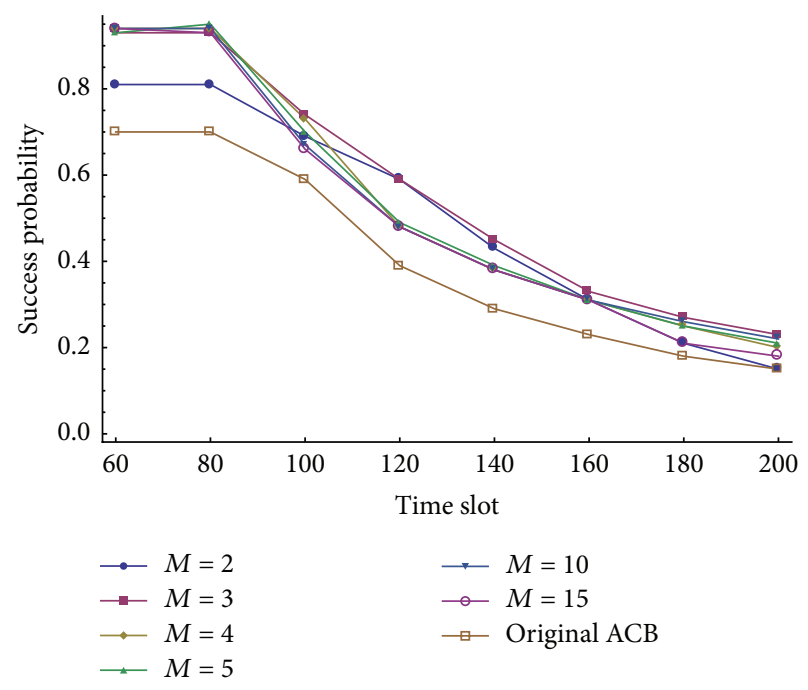

FIGURE 6: The success probability by varying time slots.

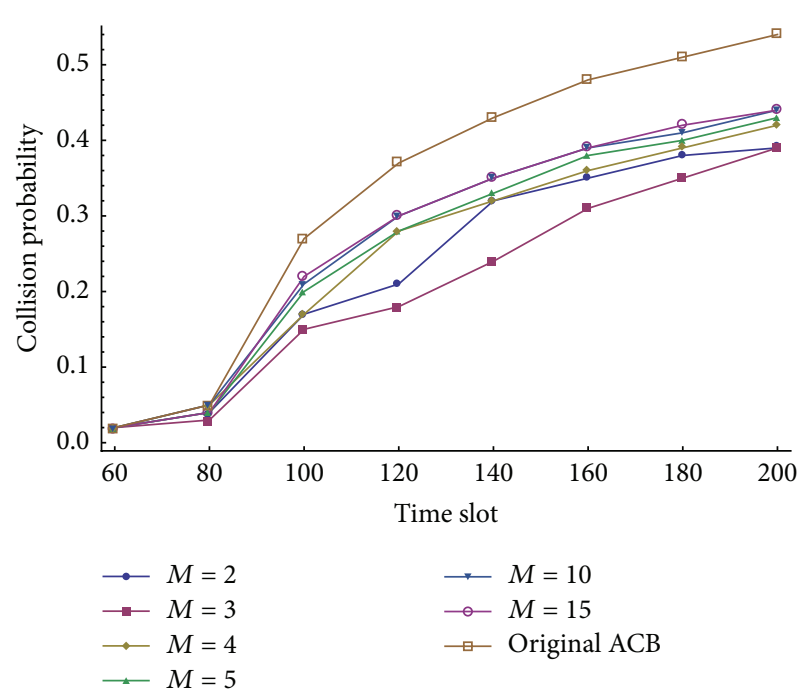

FIGURE 7: The collision probability by varying time slots.

\section{Conclusion}

In this paper, we proposed a dynamic access barring method that controls the barring factor according to the access intensity. By measuring the access intensity at the end of each time interval, we construct the transition probability among the access intensity. Then, at the end of each interval, eNB calculates the expected access intensity based on the current access intensity. Simulation results show that the proposed method outperforms the original ACB method in terms of the success probability and the collision probability because it can control the barring factor even if the traffic intensity changes over time. Simulation results also suggest that there is an optimal value for the number of access intensity levels which is the operating parameter of the proposed method. Since the number of access intensity levels influences the success probability, we leave it as a future work to devise 


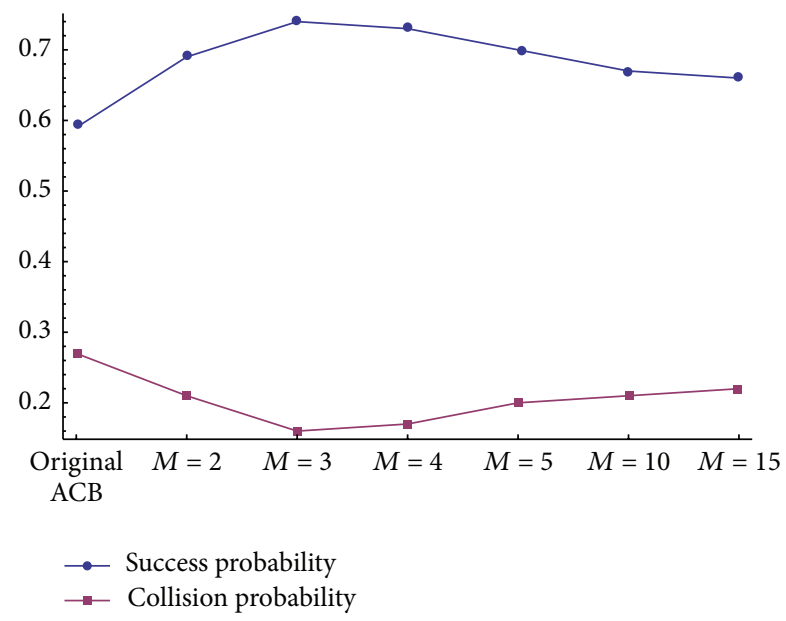

FIGURE 8: The performance by varying the number of access intensity levels.

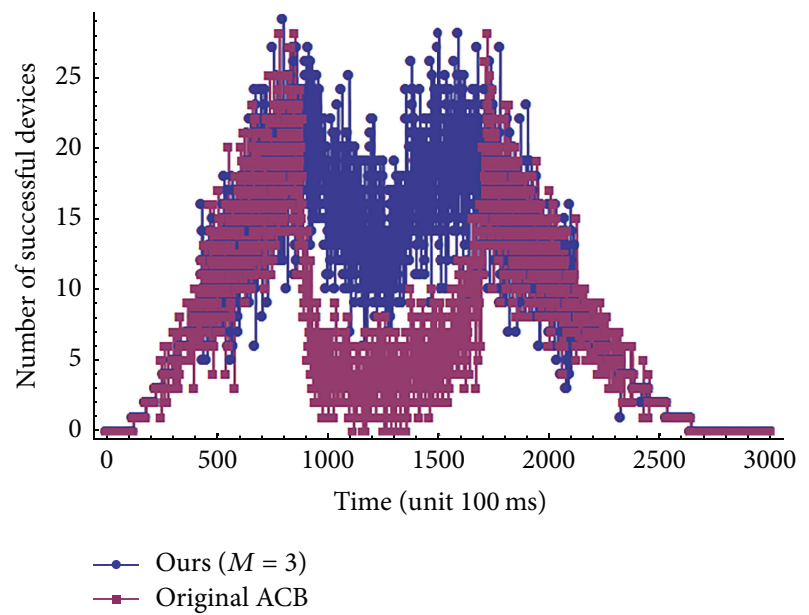

FIGURE 9: The performance comparison between our method and the original ACB.

a systematic method that finds the optimal value for the operating parameter by mathematical analysis.

\section{Competing Interests}

The authors declare that they have no competing interests.

\section{Acknowledgments}

This research was supported by the Sookmyung Women's University Research Grants (1-1603-2032) and by the Basic Science Research Program through the National Research Foundation of Korea (NRF) funded by the Ministry of Education (NRF-2015R1D1A1A01060117).

\section{References}

[1] 3rd Generation Partnership Project, Technical specification group radio access network; study on RAN improvements for machine-type communications, 3GPP TR 37.868 V11.0.0, 2011.

[2] 3rd Generation Partnership Project, "Evolved universal terrestrial radio access (E-UTRA); radio resource control (RRC); protocol specification,” 3GPP TS 36.331 V12.7.0, 2015.

[3] P. Jain, P. Hedman, and H. Zisimopoulos, "Machine type communications in 3GPP systems," IEEE Communications Magazine, vol. 50, no. 11, pp. 28-35, 2012.

[4] M. Hasan, E. Hossain, and D. Niyato, "Random access for machine-to-machine communication in LTE-advanced networks: issues and approaches," IEEE Communications Magazine, vol. 51, no. 6, pp. 86-93, 2013.

[5] M.-Y. Cheng, G.-Y. Lin, H.-Y. Wei, and A. C.-C. Hsu, "Overload control for machine-type-communications in LTE-advanced system," IEEE Communications Magazine, vol. 50, no. 6, pp. 3845, 2012.

[6] R.-G. Cheng, J. Chen, D.-W. Chen, and C.-H. Wei, "Modeling and analysis of an extended access barring algorithm for machine-type communications in LTE-A networks," IEEE Transactions on Wireless Communications, vol. 14, no. 6, pp. 2956-2968, 2015.

[7] A. Ksentini, Y. Hadjadj-Aoul, and T. Taleb, "Cellular-based machine-to-machine: overload control," IEEE Network, vol. 26, no. 6, pp. 54-60, 2012.

[8] Z. Wang and V. W. S. Wong, "Optimal access class barring for stationary machine type communication devices with timing advance information," IEEE Transactions on Wireless Communications, vol. 14, no. 10, pp. 5374-5387, 2015.

[9] S. Duan, V. Shah-Mansouri, and V. W. S. Wong, "Dynamic access class barring for M2M communications in LTE networks," in Proceedings of the IEEE Global Communications Conference (GLOBECOM '13), pp. 4747-4752, Atlanta, Ga, USA, December 2013.

[10] S. Duan, V. Shah-Mansouri, Z. Wang, and V. Wong, "D-ACB: adaptive congestion control algorithm for bursty M2M traffic in LTE networks," IEEE Transactions on Vehicular Technology, no. 99, p. 1, 2016.

[11] G. Wang, X. Zhong, S. Mei, and J. Wang, "An adaptive medium access control mechanism for cellular based machine to machine (M2M) communication," in Proceedings of the IEEE International Conference on Wireless Information Technology and Systems (ICWITS '10), pp. 1-4, Honolulu, Hawaii, USA, September 2010.

[12] S.-Y. Lien, T.-H. Liau, C.-Y. Kao, and K.-C. Chen, "Cooperative access class barring for machine-to-machine communications," IEEE Transactions on Wireless Communications, vol. 11, no. 1, pp. 27-32, 2012.

[13] H. He, Q. Du, H. Song, W. Li, Y. Wang, and P. Ren, “Trafficaware ACB scheme for massive access in machine-to-machine networks," in Proceedings of the IEEE International Conference on Communications (ICC '15), pp. 617-622, London, UK, June 2015.

[14] Verizon, Smart grid traffic behaviour discussion, 3GPP R2102340, RAN WG2 Meeting \#69b, 2010.

[15] Intel Corporation, "Further performance evaluation of EAB information update mechanisms," 3GPP R2-120270, RAN WG2 Meeting \#77, 2012. 

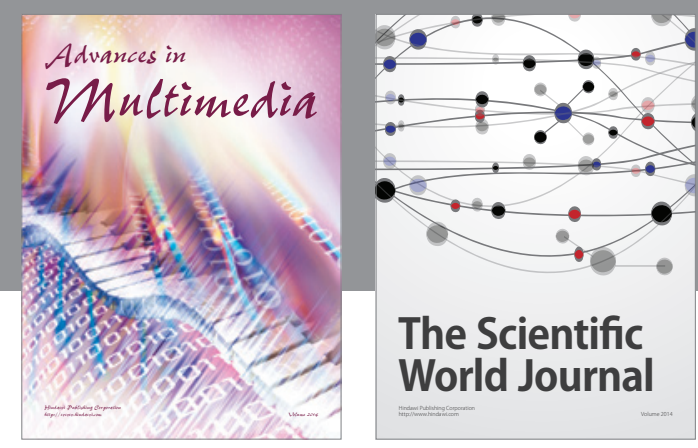

The Scientific World Journal
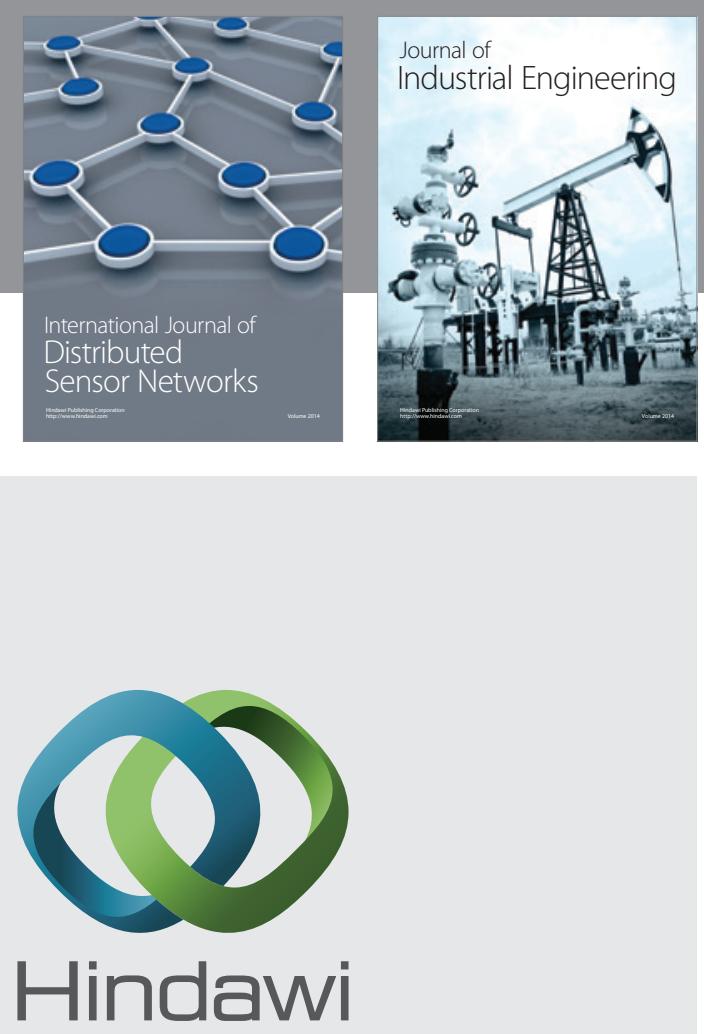

Submit your manuscripts at

http://www.hindawi.com

\section{Computer Networks} and Communications
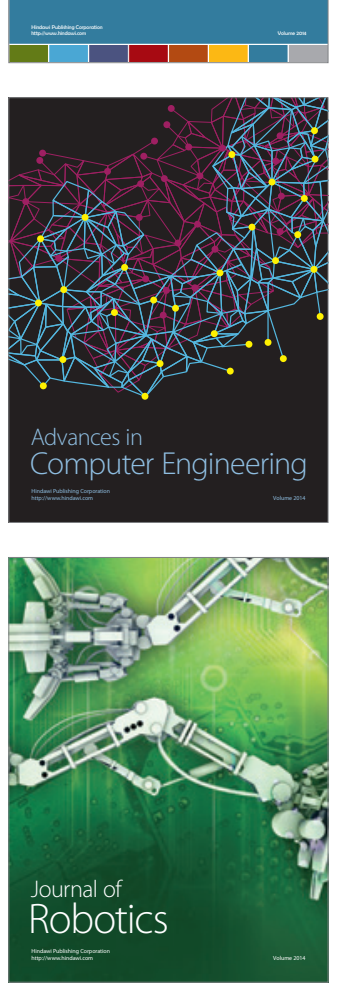
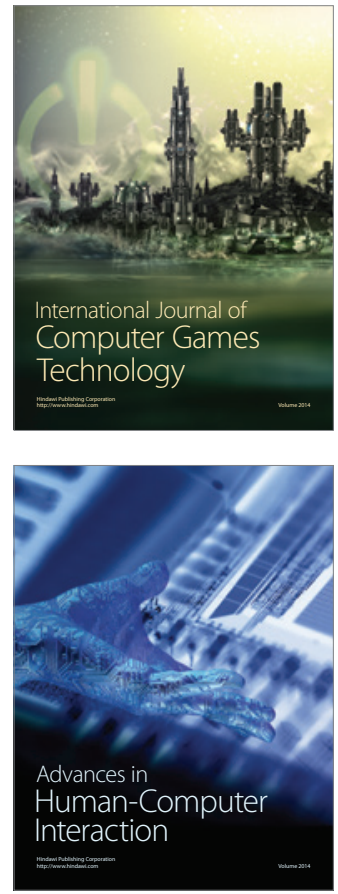
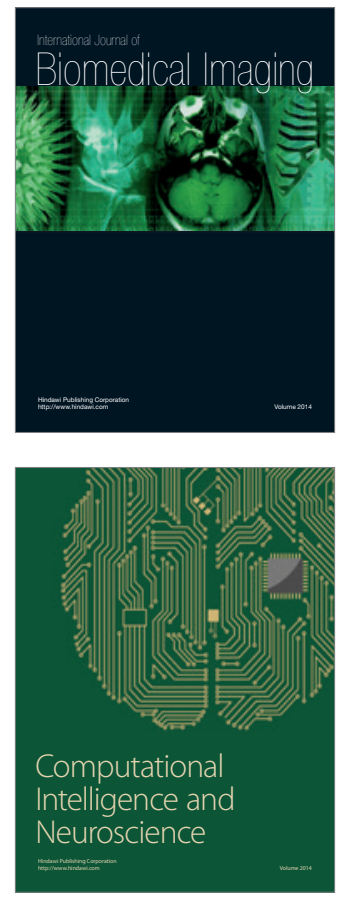
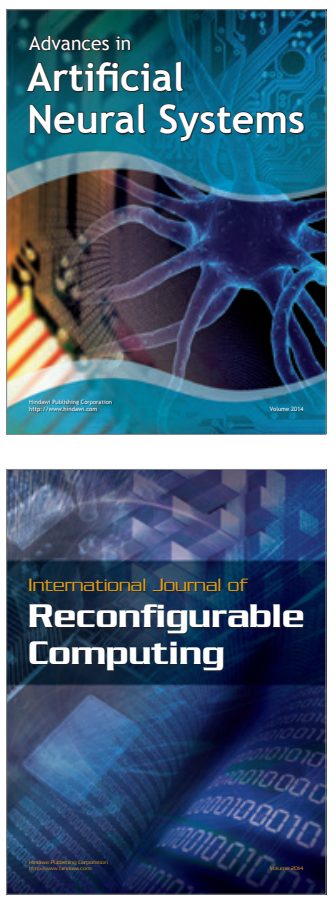
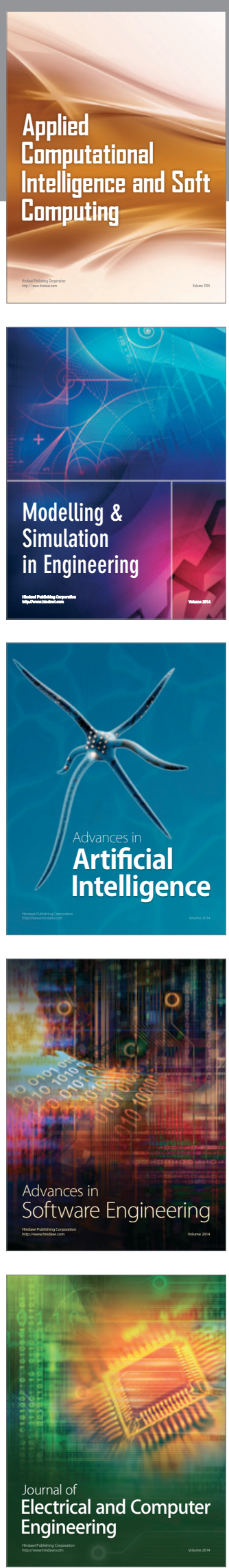